\title{
A novel higher order sliding mode control scheme $^{\star}$
}

\author{
Michael Defoort ${ }^{*, a}$ Thierry Floquet ${ }^{\mathrm{b}}$ Annemarie Kokosy $^{\mathrm{c}}$ \\ Wilfrid Perruquetti ${ }^{b}$ \\ ${ }^{a}$ Department of System Design Engineering, Keio University, Yokohama, Japan \\ ${ }^{\mathrm{b}}$ Lagis, Ecole centrale de Lille, Cité Scientifique, 59651 Villeneuve d'Ascq, France \\ INRIA Lille Nord Europe, Alien project, Parc scientifique de la Haute Borne, \\ 59650 Villeneuve d'Ascq, France \\ ${ }^{\mathrm{c}}$ Lagis, ISEN, 41 Boulevard Vauban, 59046 Lille, France
}

\begin{abstract}
A higher order sliding mode control algorithm is proposed for a class of uncertain multi-input multi-output nonlinear systems. This problem can be viewed as the finite time stabilization of a higher order input-output dynamic system with bounded uncertainties. The developed control scheme is based on geometric homogeneity and sliding mode control. The proposed procedure provides explicit conditions on the controller parameters and guarantees robustness against uncertainties. An illustrative example of a hovercraft vessel control demonstrates the advantages of the strategy.
\end{abstract}

Key words: Finite time stability, higher order sliding mode control, Geometric homogeneity.

\section{Introduction}

Sliding mode control (SMC) [28] is a robust technique to control nonlinear systems operating under uncertainty conditions $[5,7,24]$. The conventional SMC design approach consists of two steps. First, a sliding manifold is designed such

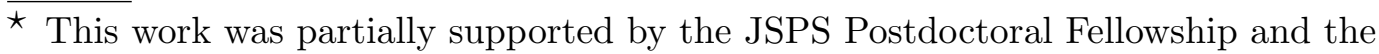
FEDER (European Funds of Regional Development) under the ARCIR Robocoop and the AUTORIS-TAT T31 projects.

* Corresponding author. E-mail: michael.defoort@gmail.com 
that the system trajectories along the manifold meet the desired performance. Then, a discontinuous control is designed such that the system trajectories reach and stay, after a finite time, on the manifold. In spite of claimed robustness properties, the resulting controller has a specific disadvantage. Its drawback is the chattering phenomenon, i.e. high frequency vibrations of the controlled system, which degrades the performance and may lead to instability.

Preserving the main advantages of the conventional SMC, an approach, called higher order $S M C$, has been proposed in order to reduce the chattering phenomenon $[6,8,11]$. Instead of influencing the first sliding variable time derivative, the signum function acts on its higher order time derivative. This method can also achieve a better accuracy with respect to discrete sampling time than conventional SMC [11].

Many papers are available in the case of second order SMC $[1,10,11]$. Arbitrary order sliding mode controllers have been recently proposed in [16-20]. The algorithm proposed in [18] allows the higher order sliding mode by tuning only one gain parameter. However, the convergence rate cannot be arbitrarily selected. One of the main problems of algorithms $[19,20]$ is parameter adjustment. Indeed, although the control parameters may be obtained using the proofs, no explicit condition for the gain tuning is derived. Hence, the convergence cannot be easily made arbitrarily fast or slow. The approach given in [16] combines SMC with linear quadratic one. In spite of several advantages (constructive approach, practical applicability), this approach suffers of a major drawback: the higher order sliding mode is only practical, i.e. the control ensures that the system trajectories reach an arbitrary small neighborhood of the origin in finite time. In [17], the controller is based on integral SMC [27] and guarantees the establishment of a higher order sliding mode. The advantages of this method are the easiness of its implementation and the robustness of the system during the entire response. However, two drawbacks appear in this approach: it directly depends on the initial conditions of the system (which may not be accurately known because of noised measurements) and complex off-line computations are needed before starting the control action.

The aim of this paper is to propose a new controller for which:

- the higher order sliding mode establishment is guaranteed in finite time,

- robustness of the system against uncertainties is ensured,

- the approach is constructive, i.e. the tuning of the control parameters is formalized,

- no complex off-line computation (for instance, resolution of an optimal control problem) is needed,

- the accurate knowledge of the initial conditions of the system is eliminated.

The problem of higher order SMC is formulated in input-output terms (as in 
[18]) through the differentiation of the sliding variable and is equivalent to the finite time stabilization of higher order integrator chains with bounded nonlinear uncertainties. Most of the finite time stabilization results available in the literature are only applicable to the second or third order integrator chain $[2,3,12]$. In [2], a robust discontinuous control scheme guarantees the finite time stabilization of a perturbed triple integrator when only the sign of the output and its first and second time derivatives are available. In the higher order dimension case, some controllers are proposed in $[4,13]$, but the finite time stabilization is not ensured if the system is perturbed. In [14], the authors give a recursive design algorithm for the construction of a global finite time controller for a class of uncertain nonlinear systems but the expression of the control input is not explicit. Here, the proposed constructive algorithm combines the finite time controller based on geometric homogeneity, which has been introduced in [4] for the finite time stabilization of an integrator chain without uncertainty, with a discontinuous controller based on SMC in order to ensure the robustness with respect to the uncertainties. This constructive procedure is especially important for higher relative degrees, when the number of control parameters is significant. Moreover, due to the large freedom and easiness of the controller, one can arbitrarily hasten or slow down the finite time transient process.

The outline of this paper is as follows. Section II states the problem and assumptions. In section III, the proposed higher order SMC is derived. In Section IV, simulations for the robust control of a hovercraft vessel demonstrate the efficiency and the advantages of the proposed algorithm compared to the procedure given in [18].

\section{Problem formulation}

Consider the following general multi-input multi-output nonlinear uncertain system:

$$
\begin{aligned}
\dot{x} & =f(x)+\sum_{i=1}^{m} g_{i}(x) u_{i} \\
y_{1} & =\sigma_{1}(x) \\
& \vdots \\
y_{m} & =\sigma_{m}(x)
\end{aligned}
$$

where $x \in \mathbb{R}^{n}$ and $u=\left[u_{1}, \ldots, u_{m}\right]^{T} \in \mathbb{R}^{m}$ are the state variable and the control input, respectively. $f(x)$ and $g(x)=\left[g_{1}(x), \ldots, g_{m}(x)\right]^{T}$ are uncertain smooth functions. $\sigma(x)=\left[\sigma_{1}(x), \ldots, \sigma_{m}(x)\right]^{T} \in \mathbb{R}^{m}$ is a smooth measurable output vector. The uncertainties on $f(x)$ and $g(x)$ are due to parameter variations, unmodeled dynamics or external disturbances and do not satisfy the 
well-known matching condition. The control objective consists in the vanishing of output $\sigma(x)$ in finite time.

Assumption 1 The relative degree vector $r=\left[r_{1}, \ldots, r_{m}\right]^{T}$ of system (1) with respect to $\sigma(x)$ is assumed to be constant and known. It means that the $m \times m$ matrix:

$$
B(x)=\left[\begin{array}{ccc}
L_{g_{1}} L_{f}^{r_{1}-1} \sigma_{1}(x) & \ldots & L_{g_{m}} L_{f}^{r_{1}-1} \sigma_{1}(x) \\
\vdots & & \vdots \\
L_{g_{1}} L_{f}^{r_{m}-1} \sigma_{m}(x) & \ldots & L_{g_{m}} L_{f}^{r_{m}-1} \sigma_{m}(x)
\end{array}\right]
$$

is nonsingular and $L_{g_{j}} L_{f}^{k} \sigma_{i}(x)=0$, for $1 \leq i \leq m, 1 \leq j \leq m$ and $0 \leq$ $k<r_{i}-1$. Moreover, it is supposed that the associated zero dynamics are asymptotically stable.

Remark 1 If the relative degree is not well defined, the addition of integrations on certain input channels can be helpful in obtaining a well defined relative degree (see [15] for further details on the Dynamic Extension Algorithm).

Definition 1 [11] Consider nonlinear system (1) and sliding variable $\sigma(x)$. Assume that the time derivatives $\sigma_{i}, \dot{\sigma}_{i}, \ldots, \sigma_{i}^{\left(r_{i}-1\right)}$ for all $i=1, \ldots, m$ are continuous functions. The manifold defined as:

$$
\mathcal{S}^{r}=\left\{x: \mid \begin{array}{c}
\sigma_{1}(x)=\dot{\sigma}_{1}(x)=\ldots=\sigma_{1}^{\left(r_{1}-1\right)}(x)=0 \\
\vdots \\
\sigma_{m}(x)=\dot{\sigma}_{m}(x)=\ldots=\sigma_{m}^{\left(r_{m}-1\right)}(x)=0
\end{array}\right\}
$$

is called the $r^{\text {th }}$ order sliding set. If it is non empty and locally an integral set in the Filippov sense [9], the motion on $\mathcal{S}^{r}$ is called $r^{\text {th }}$ order sliding mode with respect to the sliding variable $\sigma$.

The $r^{\text {th }}$ order SMC approach allows the finite time stabilization of each variable $\sigma_{i}$ and its $r_{i}-1$ first time derivatives by defining a suitable discontinuous control law. The $r_{i}^{\text {th }}$ time derivative of each function $\sigma_{i}$ yields:

$$
\left[\sigma_{1}^{\left(r_{1}\right)}(x), \ldots, \sigma_{m}^{\left(r_{m}\right)}(x)\right]^{T}=A(x)+B(x) u
$$

with

$$
A(x)=\left[L_{f}^{r_{1}} \sigma_{1}(x), \ldots, L_{f}^{r_{m}} \sigma_{m}(x)\right]^{T} .
$$

Assumption 2 Solutions of the state differential equation (2) with discontinuous right-hand side are defined in the sense of Filippov [9]. 
Assumption 3 Vector $A(x)$ and matrix $B(x)$ :

$$
\left\{\begin{array}{l}
A(x)=\bar{A}(x)+\Delta_{A}(x) \\
B(x)=\bar{B}(x)+\Delta_{B}(x)
\end{array}\right.
$$

are partitioned into nominal part (i.e. $\bar{A}$ and $\bar{B}$ ), known a priori, and uncertain bounded functions $\Delta_{A}$ and $\Delta_{B}$. Matrix $\bar{B}$ is non-singular. Furthermore, there are an a priori known nonlinear function $\rho(x)$ and an a priori known constant $1 \geq \alpha>0$ such that the uncertain functions satisfy the following inequalities:

$$
\begin{cases}\left\|\Delta_{A}(x)-\Delta_{B}(x) \bar{B}^{-1}(x) \bar{A}(x)\right\| & \leq \rho(x) \\ \left\|\Delta_{B}(x) \bar{B}^{-1}(x)\right\| & \leq 1-\alpha\end{cases}
$$

for $x \in \mathcal{X} \subset \mathbb{R}^{n}, \mathcal{X}$ being an open subset of $\mathbb{R}^{n}$ within which the boundedness of the system trajectories is ensured.

Let us apply to system (2) the following preliminary feedback:

$$
u=\bar{B}^{-1}\{-\bar{A}+w\}
$$

where $w=\left[w_{1}, \ldots, w_{m}\right]^{T} \in \mathbb{R}^{m}$ is the auxiliary control input. This feedback partially decouples the nominal system (i.e. without uncertainties). Thus, system (2) can be expressed as follows:

$$
\left[\sigma_{1}^{\left(r_{1}\right)}, \ldots, \sigma_{m}^{\left(r_{m}\right)}\right]^{T}=\left[I_{m}+\Delta_{B} \bar{B}^{-1}\right] w-\Delta_{B} \bar{B}^{-1} \bar{A}+\Delta_{A}
$$

where $I_{m}$ denotes the $m \times m$ identity matrix.

The $r^{\text {th }}$ order SMC of system (1) with respect to the sliding variable $\sigma$ is equivalent to the finite time stabilization of the multivariable uncertain system:

$$
\left\{\begin{array}{c}
\left\{\begin{array}{c}
\dot{z}_{1, i}=z_{2, i} \\
\vdots \\
\dot{z}_{r_{i}-1, i}=z_{r_{i}, i} \\
{\left[\dot{z}_{r_{1}, 1}, \dot{z}_{r_{2}, 2}, \ldots, \dot{z}_{r_{m}, m}\right]^{T}=\left[I_{m}+\Delta_{B} \bar{B}^{-1}\right] w-\Delta_{B} \bar{B}^{-1} \bar{A}+\Delta_{A}}
\end{array}\right.
\end{array}\right.
$$

with $1 \leq i \leq m, 1 \leq j \leq r_{i}, z_{j, i}=\sigma_{i}^{(j-1)}, z_{i}=\left[z_{1, i}, z_{2, i}, \ldots, z_{r_{i}, i}\right]^{T}$ and $z=\left[z_{1}^{T}, \ldots, z_{m}^{T}\right]^{T}$. 


\section{Design of a higher order sliding mode controller}

In practical terms, the resolution of the finite time stabilization is a delicate task which has generally been studied for homogeneous systems of negative degree with respect to a flow of a complete vector field. Indeed, for this kind of systems, finite time stability is equivalent to asymptotic stability (see $[3,4,13,21-23]$ for more details). However, the existing techniques are generally not constructive or robust with respect to uncertainties. To the authors' knowledge, a constructive feedback control law for finite time stabilization of all-dimension chain of integrators without uncertainty has only been proposed in [4]. Before designing our robust finite time controller, let us introduce the algorithm given in [4] and let us show its problem in terms of robustness.

\subsection{Finite time stabilization of an integrator chain system}

Let us consider the nominal system of (7) (i.e. $\Delta_{A}=0$ and $\Delta_{B}=0$ ), which is represented by $m$ single-input single-output independent integrator chains, defined as follows:

$$
\forall i \in\{1, \ldots, m\}, \quad\left\{\begin{array}{c}
\dot{z}_{1, i}=z_{2, i} \\
\vdots \\
\dot{z}_{r_{i}-1, i}=z_{r_{i}, i} \\
\dot{z}_{r_{i}, i}=w_{n o m, i}
\end{array}\right.
$$

The following result, introduced in [4], proves the existence of a continuous finite time stabilizing feedback controller for system (8) by giving an explicit construction involving a small parameter. This controller renders the closedloop system asymptotically stable and homogeneous of negative degree with respect to a suitable dilatation so that finite time stability follows. Note that the proof of asymptotic stability along with a continuity argument is based on the existence of a nonempty compact set that is strictly positively invariant with respect to the closed-loop vector field. One can refer to [4] for further details.

Theorem 1 (Theorem 8.1 in [4]) Let the positive constants $k_{1, i}, \ldots, k_{r_{i}, i}$ be such that polynomial $p^{r_{i}}+k_{r_{i}, i} p^{r_{i}-1}+\ldots+k_{2, i} p+k_{1, i}$ is Hurwitz. There is $\epsilon_{i} \in(0,1)$ such that, for every $\nu_{i} \in\left(1-\epsilon_{i}, 1\right)$, system (8) is stabilized at the origin in finite time under the feedback:

$$
w_{n o m, i}\left(z_{i}\right)=-k_{1, i} \operatorname{sign}\left(z_{1, i}\right)\left|z_{1, i}\right|^{\nu_{1, i}}-\ldots-k_{r_{i}, i} \operatorname{sign}\left(z_{r_{i}, i}\right)\left|z_{r_{i}, i}\right|^{\nu_{r_{i}, i}}
$$


where the standard notation sign (.) denotes the signum function and $\nu_{1, i}, \ldots, \nu_{r_{i}, i}$ satisfy:

$$
\nu_{j-1, i}=\frac{\nu_{j, i} \nu_{j+1, i}}{2 \nu_{j+1, i}-\nu_{j, i}}, j \in\left\{2, \ldots, r_{i}\right\}
$$

with $\nu_{r_{i}+1, i}=1$ and $\nu_{r_{i}, i}=\nu_{i}$.

Example 1 Theorem 1 guarantees finite time stability of the triple integrator system under the feedback:

$$
w_{\text {nom }, i}\left(z_{i}\right)=-\operatorname{sign}\left(z_{1, i}\right)\left|z_{1, i}\right|^{\frac{1}{2}}-1.5 \operatorname{sign}\left(z_{2, i}\right)\left|z_{2, i}\right|^{\frac{3}{5}}-1.5 \operatorname{sign}\left(z_{3, i}\right)\left|z_{3, i}\right|^{\frac{3}{4}}
$$

which is obtained from (9) with $r_{i}=3, k_{1, i}=1, k_{2, i}=1.5, k_{3, i}=1.5$ and $\nu_{i}=\frac{3}{4}$. Figure 1(a) depicts the evolution of the system trajectories under feedback $w_{n o m, i}\left(z_{i}\right)$. One can see that the triple integrator system is stabilized at the origin in finite time. However, when this system is perturbed, the finite time stabilization is not ensured. Indeed, considering the following uncertain system:

$$
\left\{\begin{array}{l}
\dot{z}_{1, i}=z_{2, i} \\
\dot{z}_{2, i}=z_{3, i} \\
\dot{z}_{3, i}=w_{i}+p\left(z_{i}\right)
\end{array}\right.
$$

where $p\left(z_{i}\right)=\sin \left(10 z_{1, i}\right)$ is a bounded uncertainty, the control law (10) does not guarantee finite time stability. The corresponding simulation results are shown in Fig. 1(b).

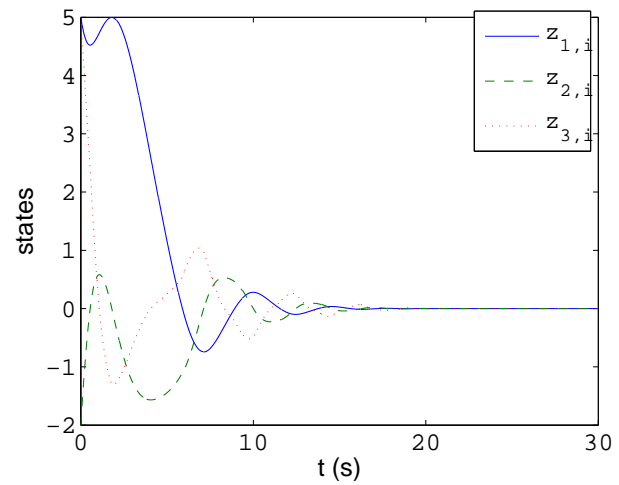

(a)

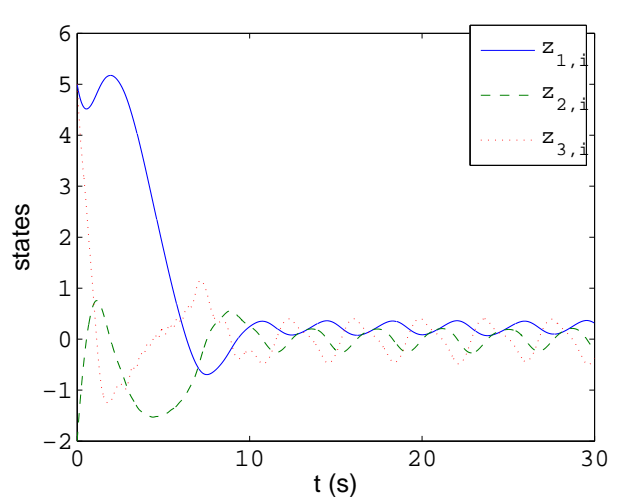

(b)

Fig. 1. Trajectories of the triple integrator system under feedback (10) (a) without perturbation (b) with pertubations.

Let us generalize the results given in [4] by designing a robust discontinuous control law which ensures the finite time stabilization of system (7). 


\subsection{Robust finite time controller design}

The higher order SMC algorithm is designed in two steps:

(1) the design of a finite time controller $w_{\text {nom }}(z)$ which guarantees the finite time stabilization of nominal system (8) at the origin,

(2) the design of discontinuous control law $w_{d i s c}(z)$ which enables to reject the uncertainties of system (7) and ensures that control objectives are fulfilled.

In order to stabilize in finite time uncertain system (7), let us define the following control law:

$$
\left\{\begin{array}{l}
w(z)=w_{\text {nom }}(z)+w_{\text {disc }}\left(z, z_{\text {aux }}\right) \\
\dot{z}_{\text {aux }}=-w_{\text {nom }}(z)
\end{array}\right.
$$

Auxiliary function $z_{\text {aux }} \in \mathbb{R}^{m}$ will be used in the design of the sliding variable associated with the discontinuous control law $w_{\text {disc }}\left(z, z_{\text {aux }}\right) \in \mathbb{R}^{m}$. The construction of control law $w_{\text {nom }}(z)=\left[w_{\text {nom }, 1}\left(z_{1}\right), \ldots, w_{\text {nom }, m}\left(z_{m}\right)\right]^{T} \in \mathbb{R}^{m}$ is given in the previous section (i.e. equation (9)).

Let us define the sliding variable $s(z) \in \mathbb{R}^{m}$, associated with $w_{\text {disc }}$, as follows:

$$
s(z)=\left[z_{r_{1}, 1}, z_{r_{2}, 2}, \ldots, z_{r_{m}, m}\right]^{T}+z_{a u x}
$$

The time derivative of $s$ along the system trajectories, can be expressed as:

$$
\begin{aligned}
\dot{s} & =\left[\dot{z}_{r_{1}, 1}, \dot{z}_{r_{2}, 2}, \ldots, \dot{z}_{r_{m}, m}\right]^{T}+\dot{z}_{\text {aux }} \\
& =\left[I_{m}+\Delta_{B} \bar{B}^{-1}\right] w-\Delta_{B} \bar{B}^{-1} \bar{A}+\Delta_{A}-w_{\text {nom }} \\
& =\left[I_{m}+\Delta_{B} \bar{B}^{-1}\right] w_{\text {disc }}-\Delta_{B} \bar{B}^{-1} \bar{A}+\Delta_{A}+\Delta_{B} \bar{B}^{-1} w_{n o m}
\end{aligned}
$$

The control law $w_{\text {disc }}$ is defined to ensure the sliding mode on $\{x \in \mathcal{X}: s=0\}$ is guaranteed in spite of uncertainties and is of the following form:

$$
w_{\text {disc }}=-G(z) \operatorname{Sign}(s)
$$

where the gain satisfies:

$$
G(z) \geq \frac{(1-\alpha)\left\|w_{\text {nom }}(z)\right\|+\rho+\eta}{\alpha}
$$

with $\eta>0$. The notation $\operatorname{Sign}\left(\left[s_{1}, \ldots s_{m}\right]^{T}\right)$ denotes $\left[\operatorname{sign}\left(s_{1}\right), \ldots, \operatorname{sign}\left(s_{m}\right)\right]^{T}$. 
Theorem 2 Consider nonlinear system (1) and assume that hypotheses 1-3 are fulfilled. Then, the control law

$$
u=\bar{B}^{-1}\left\{-\bar{A}+w_{\text {nom }}(z)+w_{\text {disc }}\left(z, z_{\text {aux }}\right)\right\}
$$

where $\dot{z}_{\text {aux }}=-w_{\text {nom }}(z), w_{\text {nom }}(z)$ and $w_{\text {disc }}\left(z, z_{\text {aux }}\right)$ are given by equations (9) and (14), respectively, ensures the establishment of a higher order sliding mode with respect to $\sigma$ in finite time.

Proof. Let us choose the following Lyapunov function:

$$
V=\frac{1}{2} s^{T} s
$$

The time derivative of $V$ along the system trajectories is expressed as:

$$
\begin{aligned}
\dot{V} & =s^{T}\left(\left[I_{m}+\Delta_{B} \bar{B}^{-1}\right] w_{\text {disc }}-\Delta_{B} \bar{B}^{-1} \bar{A}+\Delta_{A}+\Delta_{B} \bar{B}^{-1} w_{\text {nom }}\right) \\
& =s^{T}\left(-G\left[I_{m}+\Delta_{B} \bar{B}^{-1}\right] \operatorname{Sign}(s)-\Delta_{B} \bar{B}^{-1} \bar{A}+\Delta_{A}+\Delta_{B} \bar{B}^{-1} w_{\text {nom }}\right)
\end{aligned}
$$

Under the bounding relations in Assumption 3 and the choice of gain (15), one obtains:

$$
\begin{aligned}
\dot{V} & \leq-G\|s\|+(1-\alpha) G\|s\|+(1-\alpha)\left\|w_{\text {nom }}\right\|\|s\|+\rho\|s\| \\
& \leq\left(-\alpha G+(1-\alpha)\left\|w_{\text {nom }}\right\|+\rho\right)\|s\| \\
& \leq-\eta\|s\| \\
& \leq-\eta \sqrt{2} \sqrt{V}
\end{aligned}
$$

Thus, system trajectories evolve on the manifold $\{x \in \mathcal{X}: s=0\}$ in finite time and remains there in spite of the uncertainties. According to Assumption 3, matrix $I_{m}+\Delta_{B} \bar{B}^{-1}$ is uniformly invertible. In sliding mode, the equivalent control of $w_{\text {disc }}$, denoted $w_{d i s c}^{e q}$, obtained by writing $\dot{s}=0$ (see [28] for further details), is given by:

$$
w_{\text {disc }}^{e q}=\left[I_{m}+\Delta_{B} \bar{B}^{-1}\right]^{-1}\left(\Delta_{B} \bar{B}^{-1} \bar{A}-\Delta_{A}-\Delta_{B} \bar{B}^{-1} w_{\text {nom }}\right)
$$

Substituting $w=w_{n o m}+w_{d i s c}^{e q}$ into (7), one obtains the equivalent closedloop dynamics, in sliding mode, similar as the nominal system (8). Since the control law $w_{\text {nom }}$ is designed using theorem 1 , the system trajectories converge to zero in finite time. Therefore, a $r^{\text {th }}$ order sliding mode with respect to $\sigma$ is established in finite time.

Remark 2 The missing derivatives of $\sigma_{i}(1 \leq i \leq m)$ can be estimated online by means of the robust exact finite time convergent differentiator [19,26].

Example 2 In order to illustrate the proposed control strategy, let us apply theorem 2 to the perturbed triple integrator system (11). One can show that 
the origin of system (11) is stable in finite time under the control law:

$$
\begin{array}{ll}
w_{i}\left(z_{i}\right) & =w_{\text {nom }, i}\left(z_{i}\right)+w_{\text {disc }}\left(z_{i}, z_{\text {aux }}\right) \\
\dot{z}_{\text {aux }} & =-w_{\text {nom }, i}\left(z_{i}\right) \\
w_{\text {nom }, i}\left(z_{i}\right) & =-\operatorname{sign}\left(z_{1, i}\right)\left|z_{1, i}\right|^{\frac{1}{2}}-1.5 \operatorname{sign}\left(z_{2, i}\right)\left|z_{2, i}\right|^{\frac{3}{5}}-1.5 \operatorname{sign}\left(z_{3, i}\right)\left|z_{3, i}\right|^{\frac{3}{4}} \\
w_{\text {disc }}\left(z_{i}, z_{\text {aux }}\right) & =-1.5 \operatorname{sign}\left(z_{3, i}+z_{\text {aux }}\right)
\end{array}
$$

The nominal part $w_{\text {nom }, i}\left(z_{i}\right)$ is the same as the control law of example 1 . The discontinuous part, which enables to reject the perturbation $p\left(z_{i}\right)$, ensures the sliding mode on the manifold $\left\{z_{3, i}+z_{\text {aux }}=0\right\}$. In this example, $\rho\left(z_{i}\right)=1$ and $\alpha=1$. Using inequality (15), one can deduce that $G>1$. As it is theoretically expected, system trajectories are stabilized at the origin in finite time. The simulation results are depicted in Fig. 2.

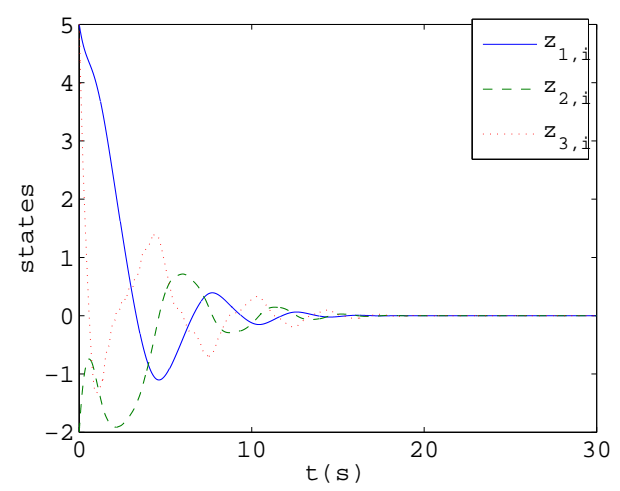

Fig. 2. Trajectories of the uncertain triple integrator system using the proposed control strategy.

\section{Higher order sliding mode control of the hovercraft vessel}

To illustrate the procedure given above, let us consider the higher order sliding mode control of a hovercraft vessel (see Fig. 3). 


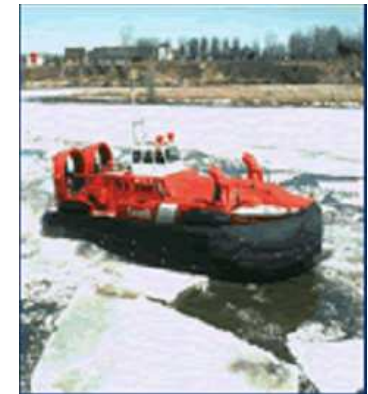

(a)

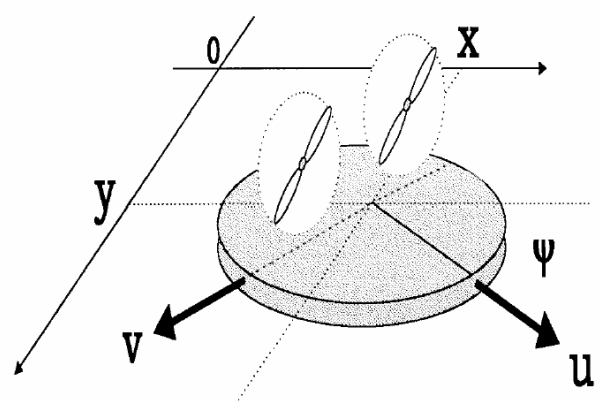

(b)

Fig. 3. Hovercraft vessel system.

\subsection{Hovercraft model}

We consider the following uncertain hovercraft model, derived in [25]:

$$
\left\{\begin{array}{l}
\dot{x}=u \cos \psi-v \sin \psi \\
\dot{y}=u \sin \psi+v \cos \psi \\
\dot{\psi}=w \\
\dot{u}=v w+\tau_{u} \\
\dot{v}=-u w-\beta v+\Delta_{v}(x) \\
\dot{w}=\tau_{w}+\Delta_{w}(t)
\end{array}\right.
$$

where $x, y$ and $\psi$ denote the position and orientation angle of the hovercraft. $u, v$ and $w$ represent its surge, sway and yaw angular velocity, respectively. $\tau_{u}$ and $\tau_{w}$ are the force and the torque applied to hovercraft. In order to test the robustness of the proposed controller, some additive perturbations are introduced in the dynamics. A matching perturbation $\Delta_{w}(t)$ affects the ship's yaw-rate dynamics. In the following simulations, $\Delta_{w}(t)$ is a gaussian noise of mean 0 and of variance 0.2 . Furthermore, in the non actuated dynamics (i.e., in the sway acceleration equation), an external perturbation force is added. It simulates a rather strong "wave field" effect and is of the form:

$$
\Delta_{v}(x)=0.15(\sin (10 x)+0.2 \cos (10 x))
$$

Assumption 4 The quantity $\beta u+\tau_{u}$ is assumed to satisfy the following inequality:

$$
\beta u+\tau_{u} \geq C>0
$$

Therefore, we are interested in maneuvers for which this quantity is bounded away from zero, which somehow limits the class of reference trajectories (see [25] for further details). 
The control objective is to design a robust control law such that the hovercraft vessel system (18) tracks the following reference trajectory:

$$
\left\{\begin{array}{l}
x_{r e f}(t)=10 \cos \left(\frac{\pi}{6} t\right) \\
y_{r e f}(t)=10 \sin \left(\frac{\pi}{6} t\right)
\end{array}\right.
$$

Let us define the following sliding variable:

$$
\sigma=\left[\begin{array}{l}
\sigma_{1} \\
\sigma_{2}
\end{array}\right]=\left[\begin{array}{l}
x-x_{r e f} \\
y-y_{r e f}
\end{array}\right]
$$

Since the relative degree of system (18) with respect to the sliding variable $\sigma$ is not well defined (the second time derivatives $\ddot{x}$ and $\ddot{y}$ depend on $\tau_{u}$ but not on $\tau_{w}$ ), a dynamic extension of system (18) is needed before designing the control. Thus, a second order integrator chain is added on the input variable $\tau_{u}$. In the following, we propose a fourth order sliding mode controller in order to solve the trajectory tracking problem.

Remark 3 In this case, the discontinuous function acts on $\left[\ddot{\tau}_{u}, \tau_{w}\right]^{T}$. Thus, high-frequency commutation of control input $\tau_{u}$ is removed. Furthermore, since the discontinuous function does not act on the first time derivative of the sliding variable (which would be the case with first order $S M C$ ), high frequency oscillations is reduced.

The fourth time derivative of $\sigma$ is:

$$
\sigma^{(4)}=\left(\bar{B}+\Delta_{B}\right)\left[\begin{array}{l}
\ddot{\tau}_{u} \\
\tau_{w}
\end{array}\right]+\left(\bar{A}+\Delta_{A}\right)
$$

with

$$
\begin{gathered}
\bar{B}=\left[\begin{array}{cc}
\cos \psi & \cos \psi(\beta v)-\sin \psi\left(\beta u+\tau_{u}\right) \\
\sin \psi & \sin \psi(\beta v)+\cos \psi\left(\beta u+\tau_{u}\right)
\end{array}\right], \Delta_{B}=\left[\begin{array}{cc}
0 & -\Delta_{v} \cos \psi \\
0 & -\Delta_{v} \sin \psi
\end{array}\right], \\
\bar{A}=\left[\begin{array}{l}
-x_{r e f}^{(4)}-w \cos \psi\left(w\left(2 \beta u+\tau_{u}\right)+2 \beta^{2} v\right)+\sin \psi\left(\beta^{3} v+w\left(\beta^{2} u-2 \dot{\tau}_{u}-\beta \tau_{u}\right)-2 w^{2} \beta v\right) \\
-y_{r e f}^{(4)}-w \sin \psi\left(w\left(2 \beta u+\tau_{u}\right)+2 \beta^{2} v\right)-\cos \psi\left(\beta^{3} v+w\left(\beta^{2} u-2 \dot{\tau}_{u}-\beta \tau_{u}\right)-2 w^{2} \beta v\right)
\end{array}\right] \\
\Delta_{A}=\left[\begin{array}{c}
\left(\Delta_{v} w^{2}+\dot{\Delta}_{v} \beta-\ddot{\Delta}_{v}-\Delta_{w} \tau_{u}-\Delta_{v} \beta^{2}-\Delta_{w} \beta u\right) \sin \psi+\left(2 \Delta_{v} \beta w+\Delta_{w} \beta v-2 \dot{\Delta}_{v} w-\Delta_{v} \Delta_{w}\right) \cos \psi \\
\left(\Delta_{w} \tau_{u}+\Delta_{v} \beta^{2}-\dot{\Delta}_{v} \beta-\Delta_{v} w^{2}+\ddot{\Delta}_{v}+\Delta_{w} \beta u\right) \cos \psi+\left(2 \Delta_{v} \beta w-\Delta_{v} \Delta_{w}-2 \dot{\Delta}_{v} w+\Delta_{w} \beta v\right) \sin \psi
\end{array}\right]
\end{gathered}
$$

$\bar{A}$ and $\bar{B}$ are the known parts whereas $\Delta_{A}$ and $\Delta_{B}$, which depend on the perturbations $\Delta_{v}$ and $\Delta_{w}$ are unknown. Under Assumption 4 , matrix $\bar{B}$ is 
uniformly invertible. Thus, using the following preliminary feedback:

$$
\left[\begin{array}{c}
\ddot{\tau}_{u} \\
\tau_{w}
\end{array}\right]=\bar{B}^{-1}(\tau-\bar{A})
$$

where $\tau \in \mathbb{R}^{2}$ is the auxiliary control input, system (20) becomes:

$$
\sigma^{(4)}=\left[I_{2}+\Delta_{B} \bar{B}^{-1}\right] \tau+\Delta_{A}-\Delta_{B} \bar{B}^{-1} \bar{A}
$$

One can note that $\left\|\Delta_{B} \bar{B}^{-1}\right\| \leq \frac{2\left|\Delta_{v}\right|}{C}$. From Theorem 2, one gets:

$$
\begin{aligned}
\tau= & \tau_{\text {nom }}+\tau_{\text {disc }} \\
\dot{z}_{\text {aux }}= & -\tau_{\text {nom }} \\
\tau_{\text {nom }}= & -24\left[\begin{array}{l}
\operatorname{sign}\left(\sigma_{1}\right)\left|\sigma_{1}\right|^{\frac{3}{7}} \\
\operatorname{sign}\left(\sigma_{2}\right)\left|\sigma_{2}\right|^{\frac{3}{7}}
\end{array}\right]-50\left[\begin{array}{l}
\operatorname{sign}\left(\dot{\sigma}_{1}\right)\left|\dot{\sigma}_{1}\right|^{\frac{1}{2}} \\
\operatorname{sign}\left(\dot{\sigma}_{2}\right)\left|\dot{\sigma}_{2}\right|^{\frac{1}{2}}
\end{array}\right]-35\left[\begin{array}{l}
\operatorname{sign}\left(\ddot{\sigma}_{1}\right)\left|\ddot{\sigma}_{1}\right|^{\frac{3}{5}} \\
\operatorname{sign}\left(\ddot{\sigma}_{2}\right)\left|\ddot{\sigma}_{2}\right|^{\frac{3}{5}}
\end{array}\right] \\
& -10\left[\begin{array}{l}
\operatorname{sign}\left(\sigma_{1}^{(3)}\right)\left|\sigma_{1}^{(3)}\right|^{\frac{3}{4}} \\
\operatorname{sign}\left(\sigma_{2}^{(3)}\right)\left|\sigma_{2}^{(3)}\right|^{\frac{3}{4}}
\end{array}\right] \\
\tau_{\text {disc }}= & -G \operatorname{Sign}\left(\sigma^{(3)}+z_{\text {aux }}\right)
\end{aligned}
$$

Term $\tau_{\text {nom }}$ is obtained by setting $r=4, k_{1}=24, k_{2}=50, k_{3}=35, k_{4}=10$ and $\nu=\frac{3}{4}$ (see Theorem 1). Gain $G$ is tuned as $G=0.2\left\|\tau_{\text {nom }}\right\|+11$ (condition (15).

\subsection{Simulation results}

Note that for the chosen reference circular trajectory, the nominal value of the quantity $\beta u+\tau_{u}$, appearing in the denominator of the controller expression, is given by:

$$
\beta u+\tau_{u}=10 \frac{\pi}{6} \sqrt{\beta^{2}+\left(\frac{\pi}{6}\right)^{2}} \neq 0
$$

The only system parameter $\beta$ is set to 1.2 . The outputs $x$ and $y$ are measured by appropriate sensors. The time derivatives of the sliding variable $\sigma$ are estimated using a higher order robust differentiator [19].

In order to highlight the advantages of the proposed controller, a comparative study between higher order SMC algorithms is carried out. Among these algorithms which allow the use of the robust differentiator (i.e. do not require the accurate knowledge of the initial high order time derivatives, up to the relative degree, of the sliding variable), do not need complex off-line computations and provide a constructive procedure for the control parameters, the 
most appealing strategy is given by the following equation [18]:

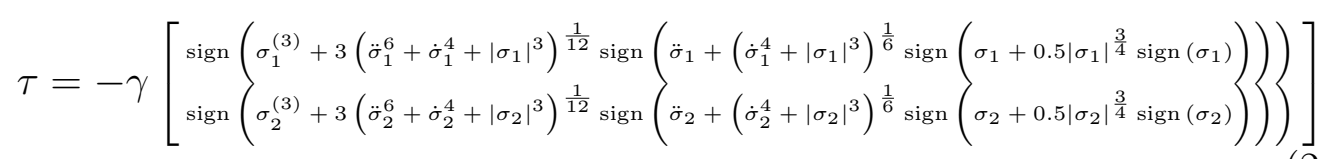

where the gain $\gamma$ is taken as $\gamma=100$.

Figures 4 and 5 show the simulation results using the proposed controller and the controller given in [18], respectively. Figures 4(a) and 5(a) depict the tracking errors $x-x_{r e f}$ and $y-y_{\text {ref }}$ and show the finite time convergence of $\sigma_{1}$ and $\sigma_{2}$ in spite that the vessel motions are started significantly far away from the reference trajectory (see Fig. 4(b) or 5(b)). Figures 4(c) and 5(c) display the corresponding surge, sway and yaw angular velocities. The two controllers efficiently correct the undesirable deviations due to the persistent perturbations. From these results, one can conclude that the chattering phenomenon is lower using the proposed strategy. Indeed, the control law [18] has only a discontinuous part. Here, the proposed controller is divided into a homogeneous part and a discontinuous one. Furthermore, contrary to [18], one can easily hasten the finite-time transient process (see the convergence rate of $\sigma_{2}$ for both algorithms). These results show the robustness and the advantages of the proposed finite time convergent controller.

\section{Conclusion}

A constructive algorithm for the design of a higher order sliding mode controller for a class of multi-input multi-output nonlinear uncertain systems is proposed. The main contribution of this paper is the design of a robust finite time convergent controller based on geometric homogeneity and sliding mode control which can be easily applied to systems with high relative degree with respect to the sliding variable. The proposed method does not require complex off-line computation and provides explicit conditions on the control parameters. A case study for finite time convergent robust control of a hovercraft vessel system is presented. Simulation results show the robustness against parameter variations and perturbation force inputs and the advantages in terms of convergence rate with respect to [18].

\section{Acknowledgements}

The authors wish to thank the anonymous reviewers for useful comments and suggestions. 


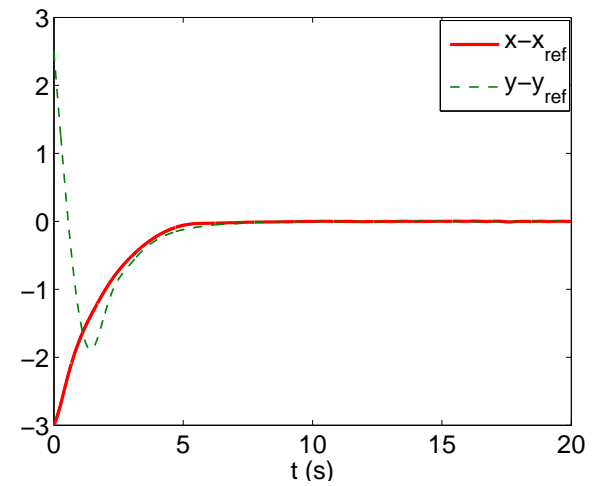

(a)

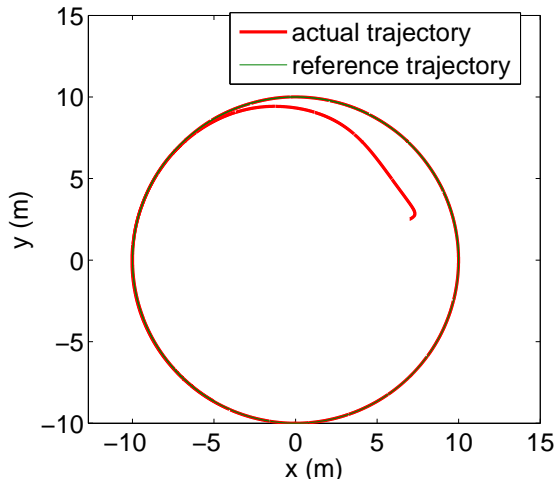

(b)

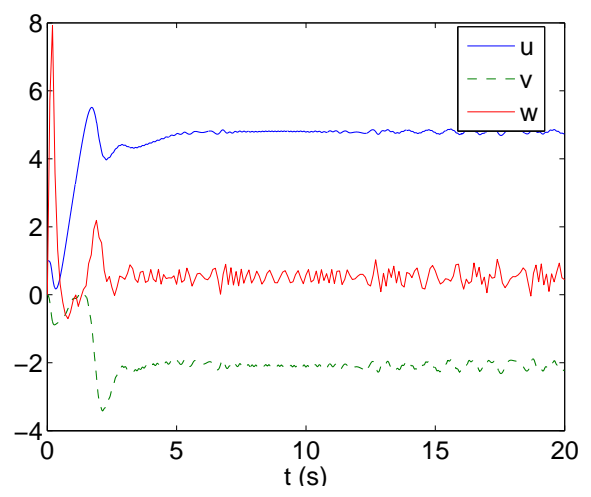

(c)

Fig. 4. Circular path tracking performance under unmodeled perturbations using the proposed algorithm.

\section{References}

[1] G. Bartolini, A. Pisano, E. Punta and E. Usai, "A survey of applications of second order sliding mode control to mechanical systems", Int. J. of Control, 76(9), pp. 875-892, 2003.

[2] G. Bartolini, A. Pisano and E. Usai, "On the finite time stabilization of uncertain nonlinear systems with relative degree three", IEEE Trans. on Automatic Control, 52(11), pp. 2134-2141, 2007.

[3] S. Bhat and D. Bernstein, "Continuous finite-time stabilitization of the translational and rotational double integrators", IEEE Trans. on Automatic Control, 43(5), pp. 678-682, 1998.

[4] S. Bhat and D. Bernstein, "Geometric homogeneity with applications to finite time stability", Mathematics of Control, Signals and Systems, 17, pp. 101-127, 2005.

[5] A. Bloch and S. V. Drakunov, "Stablization and tracking in the nonholonomic integrator via sliding modes", Syst. Cont. Let., 29(2), pp. 91-99, 1996. 


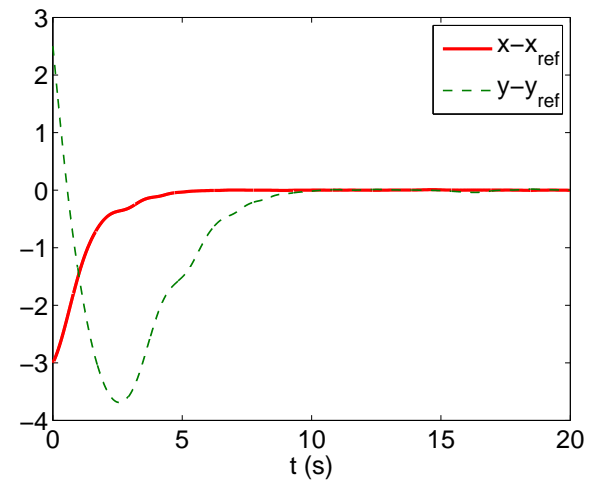

(a)

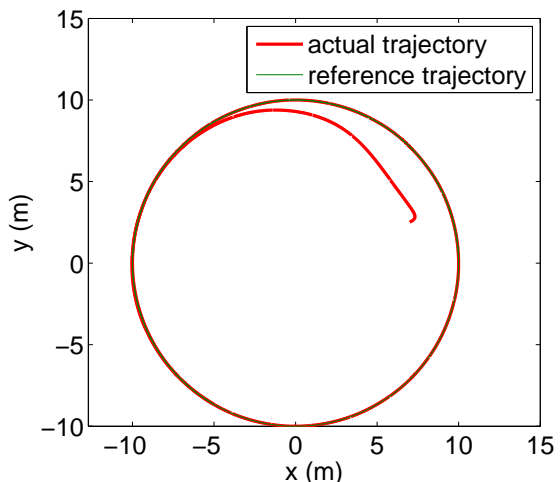

(b)

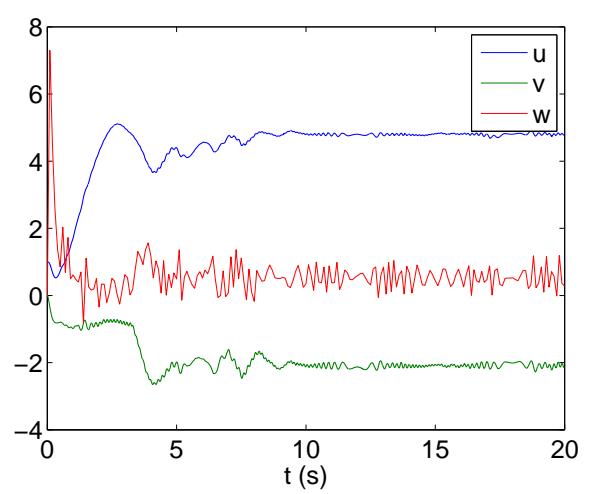

(c)

Fig. 5. Circular path tracking performance under unmodeled perturbations using the algorithm given in [18].

[6] S. V. Drakunov and V. I. Utkin, "Sliding mode control in dynamic systems", Int. J. of Control, 55(4), pp. 1029-1037, 1992.

[7] C. Edwards and S. K. Spurgeon, "Sliding mode control: theory and applications", Taylor and Francis, London, 1998.

[8] S. V. Emel'yanov, S. V. Korovin and L. V. Levantovsky, "Higher order sliding modes in control system", Differential Equations, 29(11), pp. 1627-1647, 1993.

[9] A. Filippov, "Differential equations with discontinuous right hand sides", Kluwer, Dordrecht, 1988.

[10] T. Floquet, J. P. Barbot and W. Perruquetti, "Higher order sliding mode stabilization for a class of nonholonomic perturbed systems", Automatica, 39(6), pp. 1077-1083, 2003.

[11] L. Fridman and A. Levant, "Higher order sliding modes", In W. Perruquetti and J. P. Barbot (Eds), Sliding mode control in Engineering, Marcel Dekker, New York, pp. 53-101, 2002.

[12] Y. Hong, J. Huang and Y. Xu, "On an output feedback finite-time stabilization problem", IEEE Trans. on Automatic Control, 46(2), pp. 305-309, 2001. 
[13] Y. Hong, "Finite time stabilization and stabilizability of a class of controllable systems", Syst. Cont. Let., 46(4), pp. 231-236, 2002.

[14] X. Huang, W. Lin and B. Yang, "Global finite time stabilization of a class of uncertain nonlinear systems", Automatica, 41(5), pp. 881-888, 2005.

[15] A. Isidori, Nonlinear Control Sytems II, Springler-Verlag, London, 1999.

[16] S. Laghrouche, M. Smaoui, F. Plestan and X. Brun, "igher order sliding mode control based on optimal approach of an electropneumatic actuator", Int. J. of Control, 79(2), pp. 119-131, 2006.

[17] S. Laghrouche, F. Plestan and A. Glumineau, "Higher order sliding mode control based on integral sliding mode", Automatica, 43(3), pp. 531-537, 2007.

[18] A. Levant, "Universal SISO sliding-mode controllers with finite time convergence", IEEE Trans. on Automatic Control, 46(9), pp. 1447-1451, 2001.

[19] A. Levant, "Higher-order sliding modes, differentiation and output-feedback control", Int. J. of Control, 76(9), pp. 924-941, 2003.

[20] A. Levant, "Quasi-continuous high-order sliding-mode controllers", IEEE Trans. on Automatic Control, 50(11), pp. 1812-1816, 2005.

[21] E. Moulay and W. Perruquetti , "Finite time stability and stabilization of a class of continuous systems", J. Math. Analysis Applications, 323, pp. 1430$1443,2006$.

[22] E. Moulay, M. Dambrine, N. Yeganefar and W. Perruquetti , "Finite time stability and stabilization of time delay systems", Syst. Cont. Let., 57(7), pp. 561-566, 2008.

[23] E. Moulay and W. Perruquetti , "Finite time stability of non autonomous non linear systems", Int. J. of Control, 81(5), pp. 797-803, 2008.

[24] W. Perruquetti and J. P. Barbot, "Sliding mode control in engineering", Control Eng. Series, Marcel Dekker, New York, 2002.

[25] H. Sira-Ramirez, "Dynamic second order sliding mode control of the hovercraft vessel", IEEE Trans. on Control Systems Technology, 10(6), pp. 860-865, 2002.

[26] Y. B. Shtessel and I. A. Shkolnikov, "Aeronautical and space vehicule control in dynamic sliding manifold", Int. J. of Control, 76(9), pp. 1000-1017, 2003.

[27] V. I. Utkin and J. Shi, "Integral sliding mode in systems operating under uncertainly conditions", Proceedings of the $35^{\text {th }}$ Conf. on Decision and Control, pp. 4591-4596, 1996.

[28] V. I. Utkin, J. Guldner and J. Shi, "Sliding modes control in electromechanical systems", London, Taylor and Francis, 1999. 